\title{
Changes of microbial spoilage, lipid-protein oxidation and physicochemical properties during post mortem refrigerated storage of goat meat
}

\begin{abstract}
Examined was the effect of post mortem refrigerated storage on microbial spoilage, lipidprotein oxidation and physicochemical traits of goat meat. Seven Boer bucks were slaughtered, eviscerated and aged for 24[t]. The Longissimus lumborum (LL) and Semitendinosus (ST) muscles were excised and subjected to 13 days post mortem refrigerated storage. The $\mathrm{pH}$, lipid and protein oxidation, tenderness, color and drip loss were determined in LL while microbiological analysis was performed on ST. Bacterial counts generally increased with increasing aging time and the limit for fresh meat was reached at day 14 post mortem. Significant differences were observed in malondialdehyde (MDA) content at day 7 of storage. The thiol concentration significantly reduced as aging time increased. The band intensities of myosin heavy chain (MHC) and troponin-T significantly decreased as storage progressed, while actin remained relatively stable. After 14 days of aging, tenderness showed significant improvement while muscle $\mathrm{pH}$ and drip loss reduced with increase in storage time.

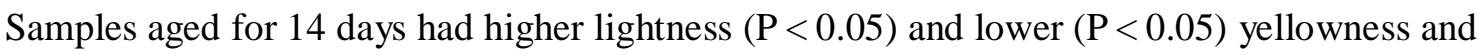
redness. Post mortem refrigerated storage influenced oxidative and microbial stability and physico-chemical properties of goat meat.
\end{abstract}

Keyword: Goat meat; Lipidï protein oxidation; Microbial spoilage; Physicochemical traits; Refrigerated storage 